\title{
RELIABILITY AND VALIDITY OF THE SEDATION SUBSCALE OF THE MODIFIED NEONATAL PAIN, AGITATION AND SEDATION SCALE (MN-PASS)
}

\author{
V. Bhatt-Mehta ${ }^{1,2}$, J.D. Barks ${ }^{2}$
}

${ }^{1}$ Clinical, Social and Administrative Sciences, College of Pharmacy, University of Michigan, ${ }^{2}$ Pediatrics, University of Michigan, Ann Arbor, MI, USA

Background: Sedation is necessary and a widely accepted practice in the mechanically ventilated (MV) neonate despite ill defined methods for measuring its depth. Benzodiazepines (BENZ) like IV Lorazepam (IVL) are used off-label as sedatives. The dosing of IVL is extrapolated from adults/older children. In animals, BENZ produce dose related neurotoxicity. Similar toxicity can be expected in the human brain with inappropriate dosing.

Aim:

1) test Reliability of the mN-PASS;

2) test Validity of the mN-PASS against the Sedation Behavior Scale(SBS) used as gold standard to measure the depth of sedation in neonates on MV.

Methods: Two trained observers scored sedation depth independently but simultaneously using the mNPASS and SBS, pre and 30 minutes post a single $0.1 \mathrm{mg} / \mathrm{kg}$ dose of IVL. Reliability and validity was evaluated using inter-rater agreement (intra class correlations (ICC)) and correlations between the two scales (Pearson's correlation), respectively.

Results: 39 neonates (GA $29.7+6.7$, PCA $39.7+6.5$, BW $1.58+1.19 \mathrm{~kg}$ ) with a total of 156 observations for each scale for the 2 observers. ICC for pre and post IVL was 0.493 to 0.78 suggesting a weak inter rater agreement. The correlation between SBS and mN-PASS was weak but significant pre and post IVL for both observers combined ( $\mathrm{r}=0.45, \mathrm{p}=0.00 ; \mathrm{r}=0.34, \mathrm{p}=0.002$; respectively) with similar trend for separate data analysis for each observer.

Conclusion: mN-PASS may not reliably assess the depth of sedation in neonates on MV. Newer scales for measuring sedation depth are needed.

Support: NIH UL1RR024986 